Zeitschrift für deutsches, europäisches

\title{
Die Aktiengesellschaft
}

und internationales Aktien-, Unternehmensund Kapitalmarktrecht

Herausgegeben von Prof. Dr. Heinz-Dieter Assmann und Prof. Dr. Mathias Habersack

die-aktiengesellschaft.de

in Verbindung mit VorsRiBGH Prof. Dr. Ingo Drescher · Prof. Dr. Volker Emmerich ·

PräsBVerfG Prof. Dr. Stephan Harbarth · Prof. Dr. Jens Koch · Prof. Dr. Hans-Joachim Mertens ·

Prof. Dr. Dr. h.c. Uwe H. Schneider · RA Prof. Dr. Jochen Vetter · Prof. Dr. Dr. h.c. Wolfgang Zöllner

\section{Jahresregister 2021}

66. Jahrgang 